\title{
UN MÉTODO ALTERNATIVO PARA DEMOSTRAR EL TEOREMA DEL LÍMITE CENTRAL
}

\author{
Wilfredo Domínguez Cirilo
}

\begin{abstract}
RESUMEN.- Presentamos una demostración alternativa del Teorema del Límite Central basado en la Regla de L'Hospital de la teoría de los límites indeterminados. El método tradicional de prueba es suponer que la variable aleatoria $X$ con distribución cualesquiera $f(x)$ posee función generatriz de momentos $m_{x}(t)$ y que además tiene media $\mu$ y varianza $\sigma^{2}$ ambas finitas. La demostración de este teorema es fundamental tanto en la estadística matemática, como en la aplicada.
\end{abstract}

PALABRAS CLAVES: Función generatriz de momentos, formas indeterminadas, serie de Taylor, esperanza y varianza.

\section{INTRODUCCION}

Usualmente en los cursos introductorios de probabilidad y estadística matemática se demuestra el Teorema del Límite Central usando la función generatriz de momentos (John E. Freud/Ronald E. Walpole 1990, pp 281-284), más precisamente, dicho teorema afirma que dada $f(x)$ la cual representa una función de densidad de probabilidades (o una función de cuantía de probabilidad) con media $\mu$ y varianza $\sigma^{2}$ ambas finitas, y $\bar{x}$ la media de una muestra aleatoria de tamaño $n$ extraida de esta población. Entonces la variable aleatoria estandarizada

$$
Z=\frac{\bar{x}-\mu}{\sigma} \sqrt{n}
$$

se aproxima a una distribución normal con media 0 y varianza 1 cuando $n$ crece indefinidamente.

Lo más notable de este teorema; es que no dice nada acerca de la forma de la función de densidad, $f(x)$. Cualesquiera sea la distribución de la v.a. con el único requisito que tenga varianza finita, para muestras grandes la distribución de la media muestral tendrá aproximadamente una distribución normal.

La importancia de este teorema, en la estadística aplicada, se debe al hecho que la media de una muestra aleatoria procedente de cualquier distribución, de probabilidades se distribuye aproximadamente como una normal con media $\mu$ y varianza $\frac{\sigma^{2}}{n}$. La demostración de este teorema requiere el uso de herramientas matemáticas avanzadas. 
Una suposición ligeramente restrictiva consiste en asumir que la variable aleatoria $X$ tiene función generatriz de momentos. La idea es averiguar si la función generatriz de momentos de la media muestral tiende a la función generatriz de momentos de una distribución normal estándar.

Es conocido que si $Z$ tiene distribución normal con media 0 y varianza 1 entonces la función generatriz de momentos es dada por

$$
m_{z_{1}}(t)=\exp \left(-\frac{t^{2}}{2}\right)
$$

Si la variable aleatoria $X$ tiene una distribución cualquiera $f(x)$, con media $\mu$ y varianza $\sigma^{2}$, y por hipótesis, posee una función generatriz de momentos, entonces la función generatriz de momentos de la variable aleatoria estandarizada,

$$
Z=\frac{X-\mu}{\sigma}
$$

sera representada por $m_{z_{2}}(t)$

La función generatriz de momentos de $Z=\frac{\bar{x}-\mu}{n} \sqrt{n}$ denotada por $m_{z_{3}}(t)$ se puede expresar en términos de $m_{z_{2}}(t)$ mediante la igualdad

$$
m_{z_{3}}(t)=\left[m_{z_{2}}\left(\frac{t}{\sqrt{n}}\right)\right]^{n}
$$

El método usual consiste en probar que, cuando $n$ tiende a infinito, el límite de la expresión del lado izquierdo de la igualdad anterior es, $\exp \left(\frac{t^{2}}{2}\right)$ Escribiremos $m_{z_{2}}(t)=p(t)+r(t)$, donde $p(t)$ es un polinomio de segundo grado en el desarrollo de la serie de Taylor para $m_{z_{1}}(t)=\exp \left(\frac{t^{2}}{2}\right)$ al rededor de 0 y $r(t)$ es el resto.

Recordando que cuando $n$ tiende a infinito, el límite de $\left(1+\frac{a}{n}\right)^{n}$ es igual a $\exp (a)$, entonces se prueba que

$$
\left[p\left(\frac{t}{\sqrt{n}}\right)+r\left(\frac{t}{\sqrt{n}}\right)\right]^{n} \rightarrow \exp \left(\frac{t^{2}}{2}\right)
$$

cuando $n$ tiende hacia el infinito. 


\section{REGLA DE L'HOSPITAL EN EL TEOREMA DEL LIMITE CENTRAL}

Consideremos las funciones uniformes $f(x)$ y $g(x)$ derivables en un entorno de $a$, tal que la derivada de $g(x)$ evaluada en $a$ no se anula. Ahora si $f(x)$ y $g(x)$ son infinitamente pequeños o infinitamente grandes cuando $x$ tiende a $\boldsymbol{a}$, es decir, la fracción $\frac{f(x)}{g(x)}$ evaluada en $x=\boldsymbol{a}$ representa una, expresión indeterminada de la forma $\frac{0}{0}, \frac{\infty}{\infty}$ entonces la regla de L'Hospital afirma que:

$$
\lim _{x \rightarrow a} \frac{f(x)}{g(x)}=\lim _{x \rightarrow a} \frac{f^{\prime}(x)}{g^{\prime}(x)}
$$

con la condición de que el límite del cociente de las derivadas, exista.

Los límites de las expresiones indeterminadás de las formas $1^{\infty}, 0^{\infty}, 0^{0}$ se determinan calculando previamente sus logaritmos y hallando el límite de la expresión $[h(x)]^{u(x)}$

Ahora probaremos el Teorema del Límite Central usando la metodología de L'Hospital.

Deseamos hallar el límite de

$$
L=\left[m_{z_{2}}\left(\frac{t}{\sqrt{n}}\right)\right]^{n}=L=\left[1+\frac{t^{2}}{2 n}+\frac{\mu_{3} t^{3}}{6 n^{3 / 2} \sigma^{3}}+\cdots\right]^{n}
$$

cuando $n$ tiende a infinito.

La expresión anterior se vuelve indeterminada de la forma $1^{\infty}$, entonces calculamos el logaritmo natural, obteniendo

$$
\begin{aligned}
\ln L & =n \ln \left[1+\frac{t^{2}}{2 n}+\frac{\mu_{3} t^{3}}{6 n^{3 / 2} \sigma^{3}}+\cdots\right] \\
& =\frac{\ln \left[1+\frac{t^{2}}{2 n}+\frac{\mu_{3} t^{3}}{6 n^{3 / 2} \sigma^{3}}+\cdots\right]}{\frac{1}{n}}
\end{aligned}
$$

Nuevamente, al tomar el límite cuando $n \rightarrow \infty$, resulta una indeterminación de la forma $\frac{0}{\infty}$. Derivamos el numerador y denominador de la igualdad anterior, con respecto a, y obtenemos 


$$
\frac{\partial \ln L}{\partial a}=\frac{1}{\left[1+\frac{t^{2}}{2 n}+\frac{\mu_{3} t^{3}}{6 n^{3 / 2} \sigma^{3}}+\cdots\right]} \frac{\frac{t^{2}}{2}\left(-\frac{1}{n^{2}}\right)+\frac{\mu_{3} t^{3}}{6 \sigma^{3}}\left(-\frac{3}{2} n^{-5 / 2}\right)+\cdots}{-\frac{1}{n^{2}}}
$$

Simplificando y tomando el límite cuando $n$ tiende a infinito resulta,

$$
\ln L=\frac{t^{2}}{2}
$$

Aplicando el exponencial a ambos miembros de la expresión anterior se obtiene

$$
L=\exp \left(\frac{t^{2}}{2}\right)
$$

La expresión anterior corresponde a la función generatriz de momentos de una distribución normal estándar con media 0 y varianza 1.

Así pues cualesquiera sea la distribución de $f(x)$, en el límite, la media muestral tendrá una distribución normal siempre que $f(x)$ tenga función generatriz de momentos, o más generalmente, siempre que $f(x)$ posea momento de segundo orden respecto al origen.

El Teorema del Límite Central se aplica tanto a distribuciones discretas como continuas. Si las funciones generatrices corresponden a distribuciones discretas, el razonamiento sería similar excepto que las integrales tendrían que ser reemplazadas por sumatorias al infinito. El grado de aproximación depende del tamaño de la muestra y de la función de densidad $f(x)$.

\section{CONCLUSIÓN}

En presente artículo se presenta una nueva forma de probar el famoso teorema del Límite Central, para ello es necesario el uso de los conceptos de límites y del cálculo diferencial.

Esta demostración requiere el supuesto de que la distribución de probabilidades tenga función generatriz de momentos. Como sabemos existen funciones de probabilidad que no poseen f. g.m. por ejemplo la distribución F de Fischer. En estos casos puede usarse la función característica definida en términos de la unidad imaginaria, esta función característica siempre existe. 


\section{BIBLIOGRAFIA}

[1] Barry, R,J. “Probabilidade: Um Curso em Nivel Intermedio". IMPA Projeto Euclides Río de Janeiro 1981.

[2] Bickel, P., Doksum, K. “Mathematical Statistics”. Holden Day, 1977.

[3] Fernández, P. J. "Introducção a Teoría das Probabilidades". Editora Universidad de Brasilia. Río de Janeiro, 1973.

[4] Hogg, R. V. and Craig, A. T. "Introduction to Mathematical Statistics". 2d edition. The Macmillan Company, New York, 1965.

[5] Ross, S. H. "Applied Probability Model with Optimization Applications". Holden-Day, San francisco, 1970.

[6] Tucker H. G. "Introducción a la Teoría Matemática de las Probabilidades y la Estadística”. Editorial Vicens-Vives. España, 1966

[7] Velleman P. F., Hoaglin D. C. “Applications, Basics, and Computing of Exploratory Data Analysis”. Duxbury Press. Boston, 1981.

[8] Venstel. H. “Théorie des Probabilités”. Editions Mir. Moscou, 1978.

[9] Wani, J.K. "Probability and Statistical Inference". Appeton Century Crofts, new York, 1971.

[10] Walpole E. R. Freund E. J., “Estadística Matemática con aplicaciones”. Prentice-Hall Hispanoamérica, S.A. 1990. 